\title{
Stereographic Methods for 3D Characterization of Dislocations
}

\author{
Ben P. Eftink ${ }^{1}$, George T. Gray ${ }^{1}$ and Stuart A. Maloy ${ }^{1}$ \\ ${ }^{1 .}$ Los Alamos National Laboratory, MST-8: Materials Science in Radiation \& Dynamic Extremes, Los \\ Alamos, NM. United States of America
}

A computer program has been developed to extract three dimensional information of dislocations, interfaces, and precipitates/cavities from two transmission electron microcopy (TEM) images. This includes incorporation of crystallographic information such as dislocation line directions, dislocation slip planes and interface plane normals. Unlike the current electron tomographic methods such as weighted back projection [1,2], these computer generated tomographic models are based on stereomicroscopy [3]. While current electron tomographic methods use tens of images, stereomicroscopy only requires two.

Previous limitations of stereomicroscopy applied to TEM require a stereo-viewer or viewing by anaglyph $[4,5]$. An additional limitation was the necessity to maintain constant diffraction contrast conditions [4]. With computer aided calculations, these obstacles to stereo-microscopy have been overcome and 3D models are created. Though using different diffraction conditions to create the models introduces an error due to shifting of where the dislocation appears in the images; in most instances this is not detrimental.

An example is shown creating a model of dislocations and a $\Sigma 3$ twin boundary in an Inconel 690 sample in Figs. 1 and 2. In Fig. 1, the two images used to produce the model are presented, crystallographic directional information from diffraction patterns for both sides of the twin boundary are also incorporated with the software. The two images have different diffraction contrast conditions and are $29.5^{\circ}$ apart in tilt. The created model is presented in Fig. 2 and is shown from perspectives ranging from tilt values of $60^{\circ}$ to $60^{\circ}$.

Using the incorporated crystallographic information, it was determined that the dislocations to the left of the twin boundary slip on the $(\overline{1} 11)_{M}$ plane and have line directions near [101], while those on the right side of the interface slip on the $(111)_{\mathrm{T}}$ plane and have line directions near $[\overline{1} 2 \overline{1}]_{\mathrm{T}}$. Subscripts $\mathrm{M}$ and $\mathrm{T}$ denote matrix and twin, and were assigned to the left and right grains, respectively. The interface plane normal was also determined and is the $(\overline{1} 11)_{\mathrm{T}, \mathrm{M}}$ plane.

This new approach to utilizing stereomicroscopy opens new opportunities for combining electron tomography with in situ experiments such as straining or heating. Previous methods that utilize tens of images to create a tomogram do not feasibly combine with in situ experiments due to the time necessary to acquire the images between events. Another advantage, in the case of in situ strain experiments, which typically limit the specimen tilt to one axis is the ability to construct a tomogram using different diffraction conditions. The model presented in Fig. 2, including crystallographic information, was obtained using a single tilt axis showing the feasibility of using during an in situ straining experiment. Introduction of this method for computer generated 3D models of microstructural features observed with TEM is suited well to reduce the time, effort and imaging condition requirements from previously utilized methods [7]. 


\section{References:}

[1] S. Bals et al, C. R. Phys. 15(2-3) (2014), p. 140.

[2] G.S. Liu et al, Mater. Charact. 87 (2014), p. 1.

[3] J.F. Nankivell, Optik 20(4) (1963), p. 171.

[4] L.A. Jacome, G. Eggeler, A. Dlouhy, Ultramicroscopy 122 (2012), p. 48.

[5] B. Hudson, M.J. Makin, Journal of Physics E: Scientific Instruments 3(4) (1970), p. 311.

[6] P. Ramachandran, G. Varoquaux, IEEE Computing in Science \& Engineering 13(2) (2011), p. 40.

[7] The DOE-NE Fuel Cycle R\&D program is acknowledged for providing funding for this research.

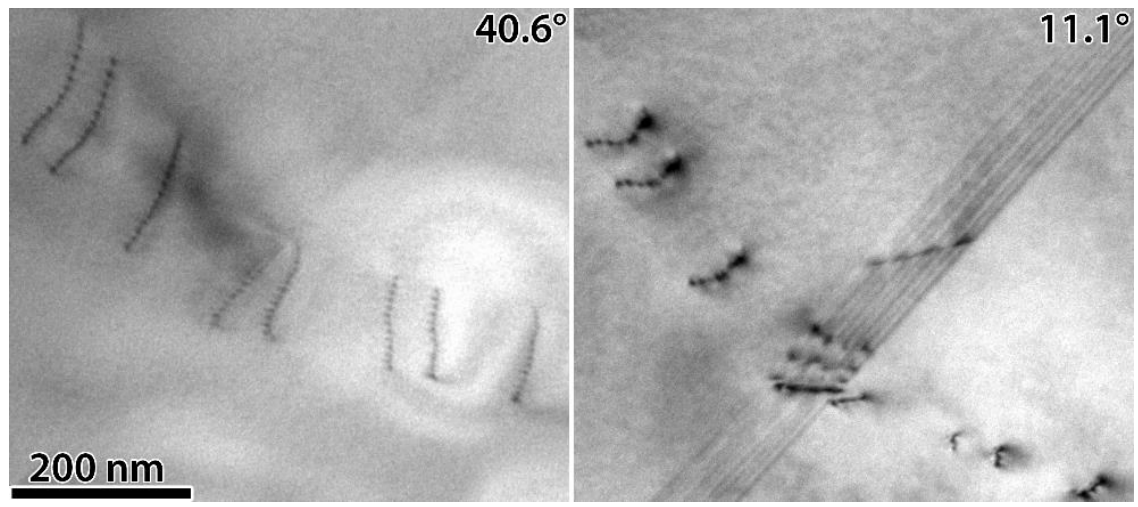

Figure 1. TEM bright-field images taken at the same location using different sample tilts.

(a)

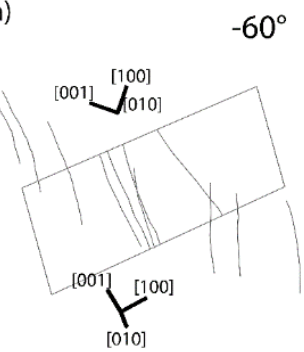

(d) (b)

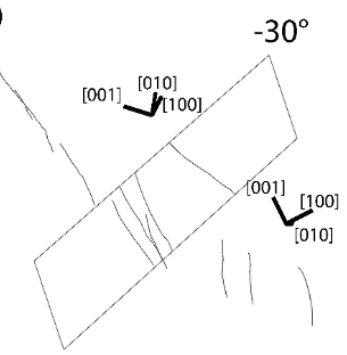

(e) (c) $0^{\circ}$

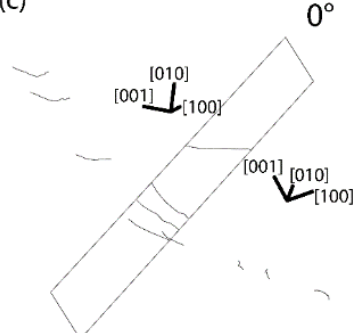

$60^{\circ}$

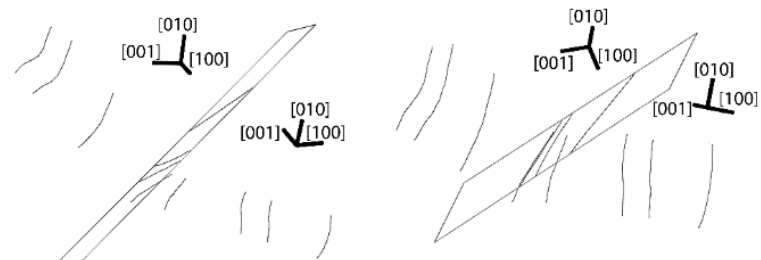

Figure 2. Computer generated model of dislocations and an interface calculated from the images in Fig. 1. Model viewed from a perspective of $-60^{\circ}$ to $60^{\circ}$ of tilt as labeled. Crystallographic directions also marked. Data visualized with Mayavi [6]. 